Gesnerus 53 (1996) 297-302

\title{
Contents of Vol. 53 (1996)
}

\section{Original Articles}

Boschung, Urs: Albrecht Haller's Patient Records (Berne 1731-1736) $\quad \ldots \ldots \ldots \ldots \ldots$

Chirila, Traian Vasile; Crawford, Geoffrey James: A Controversial Episode in the History of Artificial Cornea: The First Use of Poly(methyl methacrylate) $\quad . . \ldots \ldots \ldots \ldots \ldots$.

Dionisi, Peter: Tietze und die Zytomegalie-Erkrankung der Speicheldrüsen [Tietze and the Cytomegaly Disease of the Salivary Glands] . . . . . . . . . . . . . . . . . . . . .

Durrer, Melchior: Quantitative und qualitative Veränderungen der Mortalität zwischen 1790 und 1990 in einem Bergdorf der Zentralschweiz [Quantitative and Qualitative Changes in Mortality between 1790 and 1990 in a Swiss Mountain Village] ..........

Gelius, Rolf: Der «Processus universalis» nach Michael Sendivogius. Zur Entstehungsgeschichte einer neuzeitlichen Variante des alchimischen Grossen Werkes [Michael Sendivogius" «Processus universalis». Origins of a Modern Form of the Alchemical Work] ...

Gross, Dominik: Die Agonie des wundärztlichen Berufsstandes am Beispiel des Standespolitikers Arnold Schlegel (1850-1924) [Arnold Schlegel (1850-1924) and the Agony

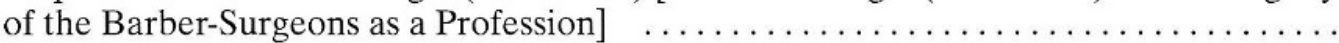

Ledermann, François: Le travail du pharmacien au début du XIX ${ }^{\mathrm{e}}$ siècle à travers une taxe de médicaments bernoise de 1815 [The Pharmacist's Work in the Early 19th Century in the Light of an 1815 Bernese Price List of Drugs] . . . . . . . . . . . . . . . . . .

Luthi, François: Le cancer est-il une maladie nouvelle? A propos du diagnostic des maladies tumorales dans le De Medicina de Celse [Is Cancer a Recent Disease? The Diagnostics of Tumors in Celsus' De Medicina $]$...............................

Lüthy, Christoph: The Life of «8K», a Vagrant Microscope Objective $\ldots \ldots \ldots \ldots \ldots$

Medicus, Heinrich A.: Heinrich Zangger und die Berufung Einsteins an die ETH. Sein Einfluss auf die Besetzung weiterer Physik-Lehrstühle in Zürich [Heinrich Zangger and Einstein's Appointment at the Swiss Federal Institute of Technology. His Influence on Other Appointments in Physics in Zurich] . ..............................

Mörgeli, Christoph; Schulz, Stefan: Die zweimalige Kaiserschnitt-Entbindung durch den Zürcher Stadtwundarzt Johann Jakob Locher von 1817/18 [Two Cesarean Sections Performed in 1817/18 on the Same Patient by the Zurich Town Surgeon, Johann Jakob

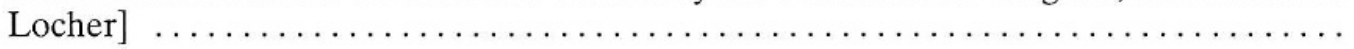


Weber, Walter: Das Herz von Frédéric de Wangen, Bischof von Basel, 1776-1782 [The Heart of Frédéric de Wangen, Bishop of Basel, 1776-1782] ....................

\section{Editorial}

Bickel, Marcel H.: 75 Jahre Schweizerische Gesellschaft für Geschichte der Medizin und der Naturwissenschaften [75th Anniversary of the Swiss Society of the History of Medi-

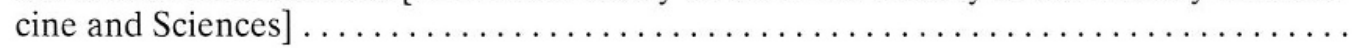

\section{Obituary}

Prof. Carl Haffter (1909-1996)

News and Activities 101,248

\section{Books Received}

\section{Book Reviews}

Essay Review: Trevisani, Francesco: Descartes in Germania (Bergdolt) …....... 105

Agrimi, Jole; Crisciani, Chiara: Les ‘Consilia’ médicaux (Seiler) . . . . . . . . . . . . 114

Ancient medicine in its socio-cultural context (B. Naef) $\ldots \ldots \ldots \ldots \ldots \ldots \ldots \ldots \ldots, 253$

Ars medica - Verlorene Einheit der Medizin? (Kubik) .................... 135

Aulbers, B. J. M.; Bremer, G. J. (eds.): De huisarts van toen (Huizink) $\ldots \ldots \ldots \ldots \ldots$

Baer, Karl Ernst von: Folia Baeriana VI: Baer and modern biology (Schmutz) _..... 274

Bardinet, Thierry: Les papyrus médicaux de l'Egypte pharaonique (S. Bickel) . . . . . . 149

Barfoot, Michael (ed.): «To ask the suffrages of the Patrons»: Thomas Laycock and the

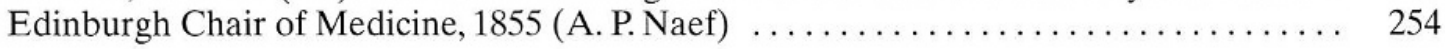

Bauer, Axel (Hrsg.): Theorie der Medizin (Raggenbass) $\ldots \ldots \ldots \ldots \ldots \ldots \ldots \ldots . \ldots \ldots$

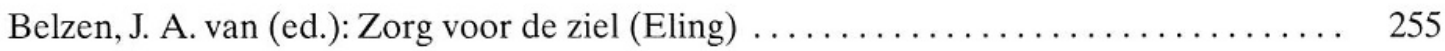

Bergdolt, Klaus: Arzt, Krankheit und Therapie bei Petrarca (Koelbing) ........... 274

Bergen, Leo van: De zwaargewonden eerst? (Haneveld) $\ldots \ldots \ldots \ldots \ldots \ldots \ldots \ldots . \ldots \ldots$

Borisov, Peter: Ginekologija na Slovenskem od začetka do leta 1980 (Levental) . . . . . 133

Boschung, Urs: Albrecht von Haller in Göttingen, 1736-1753 (Haeberli) ........... 256 
Boschung, Urs: Johannes Gessner (1709-1790) (Gantenbein) ..................

Bulletin de la Société des Sciences Médicales du Grand-Duché de Luxembourg (Kessel-

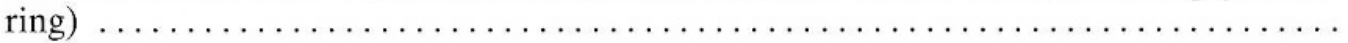

Burnett, Charles; Yamamoto, Keiji; Yano, Michio: Abü Mašar «The abbreviation of the introduction to astrology» together with the medieval Latin translation of Adelard of Bath (Gantenbein)

Carozzi, Albert V.; Bouvier, Gerda: The scientific library of Horace-Bénédict de Saussure

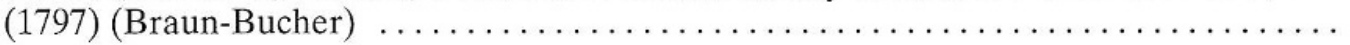

Chandrasekhar, Subrahmanyan: Newton's Principia for the common reader (Ganten-

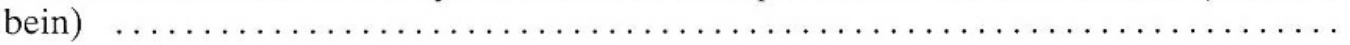

Comprendre et maîtriser la nature au moyen âge (Federici Vescovini) $\ldots . \ldots \ldots \ldots \ldots$

Corbey, Raymond; Theunissen, Bert (eds.): Ape, Man, Apeman: changing views since 1600

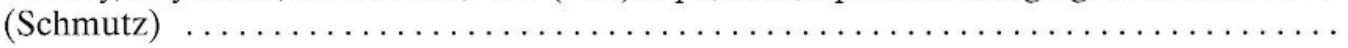

Demand, Nancy: Birth, death, and motherhood in Classical Greece (Borkowsky) .....

Dinges, Martin; Schlich, Thomas (Hrsg.): Neue Wege in der Seuchengeschichte (Ritz-

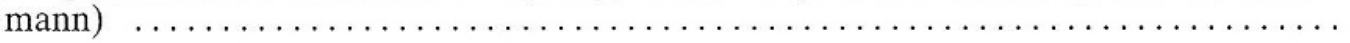

Dixon, Laurinda S.: Perilous chastity (Fischer-Homberger) $\ldots \ldots \ldots \ldots \ldots \ldots \ldots$

Einstein, Albert: The collected papers of Albert Einstein, vol. 3-5: The Swiss Years: Writings 1909-1914; Correspondence 1902-1914 (Straumann) ................... 144

Ellis, Richard H. (ed.): The case books of Dr. John Snow (Hess) $\ldots \ldots \ldots \ldots \ldots \ldots .130$

Erfüllt leben - in Gelassenheit sterben: Geschichte und Gegenwart (Baum) ....... 109

Das europäische Gesundheitssystem (Ritzmann) _..................... 277

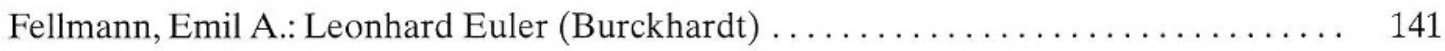

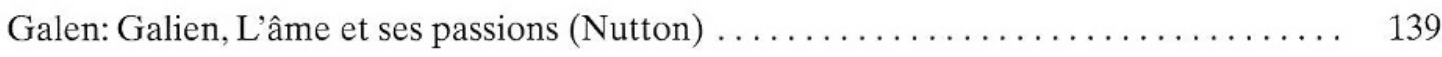

Galen: On the elements according to Hippocrates / De elementis ex Hippocratis senten-

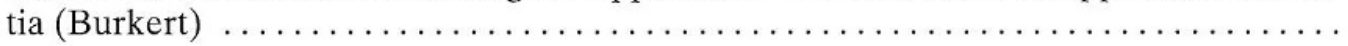

Gasser, Jacques: Aux origines du cerveau moderne. Localisations, langage et mémoire

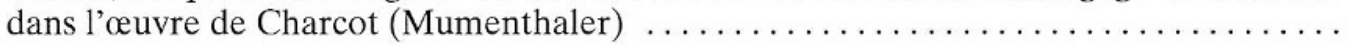

Geison, Gerald L.: The private science of Louis Pasteur (Lindenmann) $\ldots \ldots \ldots \ldots \ldots$

Gerabek, Werner: Friedrich Wilhelm Joseph Schelling und die Medizin der Romantik

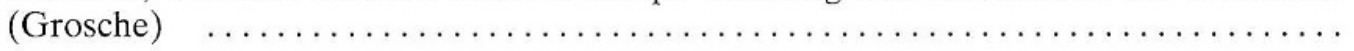

Glasser, Otto: Wilhelm Conrad Röntgen und die Geschichte der Röntgenstrahlen

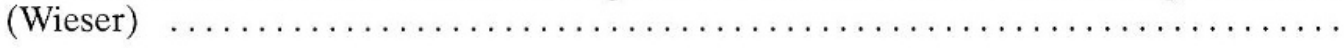

Goldammer, Kurt (Hrsg.): Paracelsus. Theologische und religionswissenschaftliche Schriften: Register (Indices) zu den Bänden IV bis VII (Gantenbein) $\ldots \ldots \ldots \ldots \ldots$ 
Grmek, Mirko D. (Hrsg.): Die Geschichte des medizinischen Denkens: Antike und

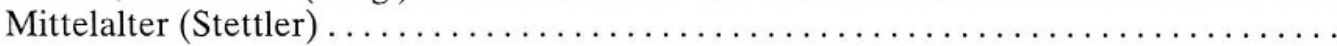

Günzel, Klaus: Die deutschen Romantiker (Grosche) $\quad \ldots \ldots \ldots \ldots \ldots \ldots \ldots \ldots \ldots$

Hagelin, Ove: Kinetic jottings: rare and curious books in the library of the old Royal Cen-

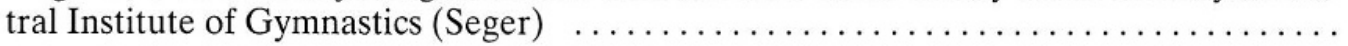

Hagenmeyer, Christa: Das Regimen Sanitatis Konrads von Eichstätt (Riha) ........

Haynal, André: La psychanalyse: 100 ans déjà ... (Müller) ................. 285

Heil und Heilung in den Religionen (Scharfetter) $\ldots \ldots \ldots \ldots \ldots \ldots \ldots \ldots \ldots \ldots \ldots$

Hentschel, Klaus and Ann M. (eds.): Physics and National Socialism (Scharf) . . . . . . 285

Heuck, Friedrich H. W.; Macherauch, Eckard (Hrsg.): Forschung mit Röntgenstrahlen

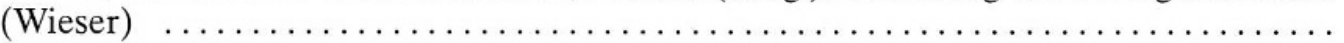

Hippocrates (von Kos): De l'art médical (Mudry) $\ldots \ldots \ldots \ldots \ldots \ldots \ldots \ldots \ldots \ldots \ldots$

Hoffmann, Dieter (Hrsg.): Gustav Magnus und sein Haus (Handel) $\ldots \ldots \ldots \ldots \ldots .146$

Hörz, Herbert: Physiologie und Kultur in der zweiten Hälfte des 19. Jahrhunderts - Briefe

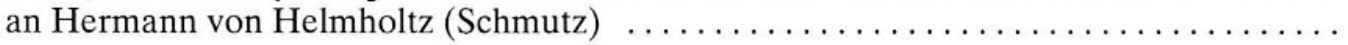

Ineichen, Robert: Würfel und Wahrscheinlichkeit. Stochastisches Denken in der Antike

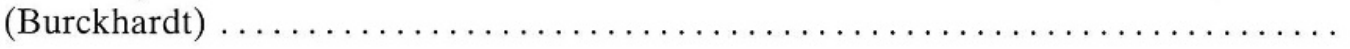

Jochmann, Carl Gustav: Briefe eines Homöopathischgeheilten an die zünftigen Wider-

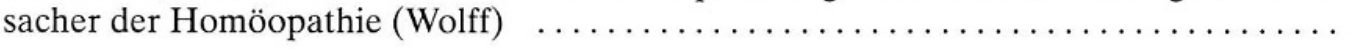

Krätz, Otto: Goethe und die Naturwissenschaften (Grosche) . . . . . . . . . . . 119

Krätz, Otto; Merlin, Helga: Casanova, Liebhaber der Wissenschaften (Grosche) ... 119

Kreuter, Alma: Deutschsprachige Neurologen und Psychiater, Band 1: Abelsdorff-Gutz-

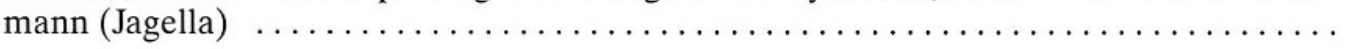

Krüger, Lorenz (Hrsg.): Universalgenie Helmholtz (Neumann) $\ldots \ldots \ldots \ldots \ldots \ldots$

Lawrynowicz, Kasimir: Friedrich Wilhelm Bessel 1784-1846 (Burckhardt) $\ldots \ldots \ldots \ldots 138$

Lebensverlängerung aus medizinischer, ethischer und rechtlicher Sicht (Renggli) $\ldots \ldots \quad 260$

Leibniz, Gottfried Wilhelm: Mathematischer, naturwissenschaftlicher und technischer

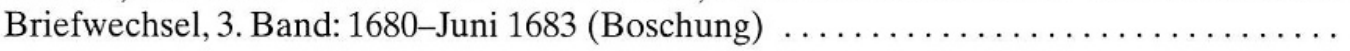

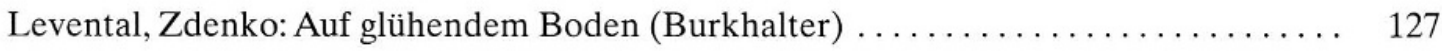

Lieburg, M. J. van: De geschiedenis van de gemeentelijke gezondheidsdienst te Rotterdam 1919-1994 (Haneveld) . . . . . . . . . . . . . . . . . . . . . . . . . . . . . . .

Lindberg, David C.: Von Babylon bis Bestiarium. Die Anfänge abendländischen Wissens

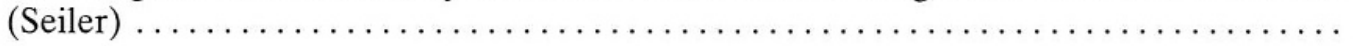


Marino, Luigi: Praeceptores Germaniae. Göttingen 1770-1820 (Hagner) ～. . . . . . . 261

Marland, Hilary; Pelling, Margaret (eds.): The task of healing (Luyendijk-Elshout) . . . . 289

Médecins érudits de Coray à Sigerist (A. P. Naef) $\ldots \ldots \ldots \ldots \ldots \ldots \ldots \ldots \ldots \ldots \ldots \ldots \ldots \ldots$

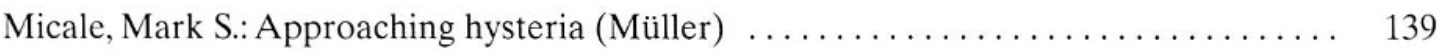

Mocek, Reinhard: Johann Christian Reil (1759-1813) (Grosche) . . . . . . . . . . . 148

Müller, Christian (éd.): Portraits de psychiatres romands (Haynal) $\ldots \ldots \ldots \ldots \ldots \ldots 263$

Müller-Tamm, Jutta: Kunst als Gipfel der Wissenschaft (Grosche) ．............ 264

Murken, Axel Hinrich; Bösing, Bernhard (Hrsg.): Medicina in nummis (Kapossy) . . . 289

Naef, Andreas P.: De la tuberculose à la greffe du cœur, 1940-1990 (Terrier) . . . . . . 265

Naturwissenschaften und Eugenik (Fischer-Homberger) $\ldots \ldots \ldots \ldots \ldots \ldots \ldots \ldots \ldots$

Neuman, William R.: The «Summa perfectionis» of Pseudo-Geber (Gantenbein) . . . . 116

Neumann, Herbert A.; Klinger, Yvonne: Knochenmark und Stammzelle (Grosche) . . . 115

Neumann, Herbert A.; Klinger, Yvonne: Knochenmark und Stammzelle (Widmer) . . . 116

Oberkof̣ler, Gerhard; Goller, Peter: Geschichte der Universität Innsbruck (1669-1945)

(Glaus) ............................................... 290

Passie, Torsten: Phänomenologisch-anthropologische Psychiatrie und Psychologie

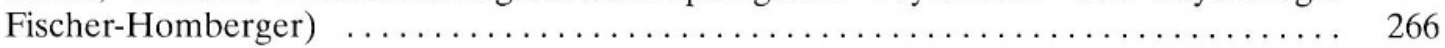

Pfister, Christian: Im Strom der Modernisierung (Schnyder) $\ldots \ldots \ldots \ldots \ldots \ldots \ldots .273$

Picherit, Jean-Louis G.: La métaphore pathologique et thérapeutique à la fin du Moyen

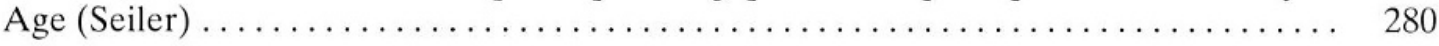

Le poids des ans. Une histoire de la vieillesse en Suisse romande (Koelbing) . . . . . . 110

Reckenfelderbäumer, Alfred: Medizin und Wissenschaftstheorie (Bickel) $\ldots \ldots \ldots \ldots 267$

Roger, Jacques: Pour une histoire des sciences à part entière (Simon) $\ldots \ldots \ldots \ldots \ldots$

Rosenmund, Klara Anna: Die Entwicklung der Klinischen Chemie in Zürich 1833-1900

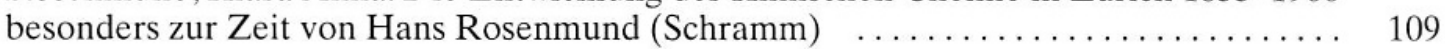

Rueb, Franz: Hexenbrände. Die Schweizergeschichte des Teufelswahns (Gantenbein) 268

Schipperges, Heinrich: Arzt im Purpur. Grundzüge einer Krankheitslehre bei Petrus

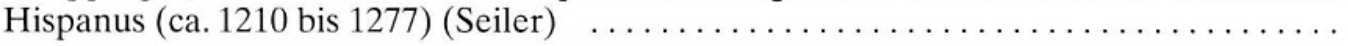

Schnalke, Thomas (Hrsg.): Natur im Bild. Anatomie und Botanik in der Sammlung des

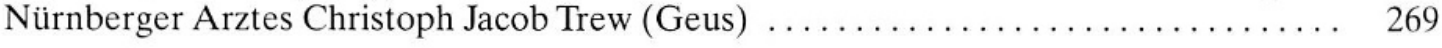

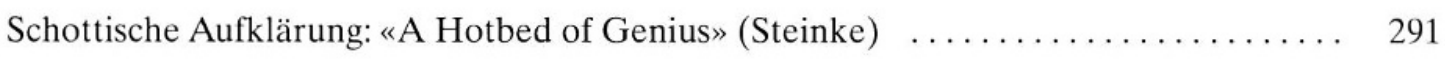

Self-motion from Aristotle to Newton (Bröer) $\ldots \ldots \ldots \ldots \ldots \ldots \ldots \ldots \ldots \ldots \ldots \ldots \ldots \ldots \ldots$ 
Spallanzani, Lazzaro: Edizione nazionale delle opere di Lazzaro Spallanzani: Lezioni,

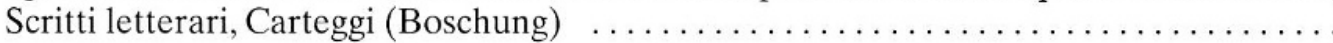

Statius van Eps, L. W.: Gezondheid op de Nederlandse Antillen: beschermd en toch be-

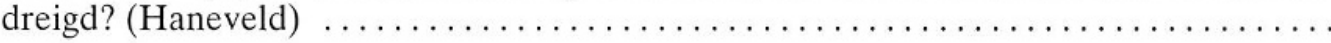

Steffens, Henrich: Was ich erlebte. Aus der Erinnerung niedergeschrieben (Hess) $\ldots . \quad 146$

Stephanus of Athens: Commentary on Hippocrates' aphorisms sections V-VI (Burkert) 134

Stoltzenberg, Dietrich: Fritz Haber, Chemiker, Nobelpreisträger, Deutscher, Jude

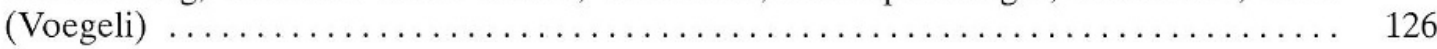

Stuber, Martin; Kraut, Sabine: Der Marcel Benoist-Preis 1920-1995 (Lindenmann) . ...

Stückelberger, Alfred: Bild und Wort. Das illustrierte Fachbuch in der antiken Natur-

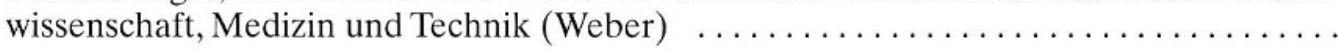

Die Technikgeschichte als Vorbild moderner Technik: Agricola-Vorträge Chemnitz 1994

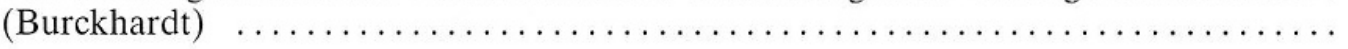

Telle, Joachim (Hrsg.): Analecta Paracelsica (Gantenbein) $\ldots \ldots \ldots \ldots \ldots \ldots \ldots \ldots$

Telle, Joachim (Hrsg.): Parerga Paracelsica: Paracelsus in Vergangenheit und Gegenwart

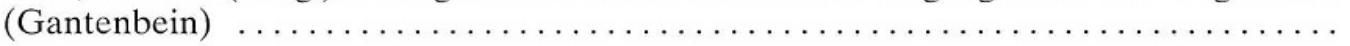

Thurston, Hugh: Early astronomy (Gantenbein) $\ldots \ldots \ldots \ldots \ldots \ldots \ldots \ldots \ldots \ldots \ldots \ldots$

Weber, Matthias M.: Ernst Rüdin (Lilienthal) $\ldots \ldots \ldots \ldots \ldots \ldots \ldots \ldots \ldots \ldots \ldots \ldots \ldots \ldots \ldots$

Weeber Karl-Wilhelm: Alltag im Alten Rom (Zimmermann) $\ldots \ldots \ldots \ldots \ldots \ldots \ldots$

Wenig, Klaus: Rudolf Virchow und Emil du Bois-Reymond. Briefe 1864-1894 (Grad-

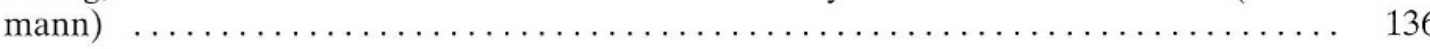

Wiesing, Urban: Kunst oder Wissenschaft? (Grosche) $\ldots \ldots \ldots \ldots \ldots \ldots \ldots \ldots \ldots . \ldots \ldots$

Windemuth, Marie-Louise: Das Hospital als Träger der Armenfürsorge im Mittelalter

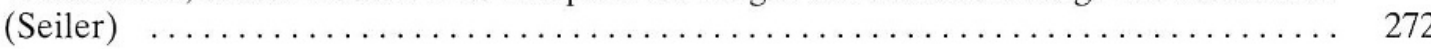

Wise, M. Norton (ed.): The values of precision (Ineichen) $\ldots \ldots \ldots \ldots \ldots \ldots \ldots \ldots, 270$

Witzler, Beate: Grossstadt und Hygiene (Gutzwiller) $\ldots \ldots \ldots \ldots \ldots \ldots \ldots \ldots \ldots$

Wolfschmidt, Gudrun: Milchstrasse - Nebel - Galaxien (Glaus) $\ldots \ldots \ldots \ldots \ldots \ldots$

Yavetz, Ido: From obscurity to enigma (Burckhardt) $\ldots \ldots \ldots \ldots \ldots \ldots \ldots \ldots \ldots \ldots$

Zamora, Juan Gil de (Johannes Aegidius Zamorensis): Historia naturalis (Geus) $\ldots . \quad 281$ 